\title{
MULTIPHONON ABSORPTION AND PHOTON AVALANCHE CRITERION IN ERBIUM DOPED
}

\section{MATERIALS}

\section{F. AUZEL}

France-telecom, CNET PAB/BAG-Laboratoire de Bagneux, Groupe Optique des Terres-rares, B.P.107, 92225, Bagneux, France

The photon avalanche effect has been recently observed at room temperature in erbium doped fluoride glasses and crystals, in a particularly clear way, displaying simultaneously all the typical features of this effect: (i) existence of a threshold for transmission at the excitation wavelength, (ii) up-conversion excitation threshold, (iii) long delay for threshold establishment. Analysing the first step of the avalanche effect as an anti-Stokes multiphonon absorption, it can be shown why this effect is so clearly observed at room temperature in our erbium studies. Our results are compared with other cases of avalanche, with cases which have been called "looping effects" and "quasi-avalanche" ones.

PACS numbers: $42.50 . \mathrm{Hz}, 42.62 . \mathrm{Fi}, 42.70 . \mathrm{IIj}, 78.50 . \mathrm{Ec}$

\section{Introduction}

The photon avalanche effect has been recently observed at room temperature in crbium doped fluoride glasses [1-4] and crystals [5], in a particularly clear way, displaying simultaneously all the typical features of this effect: (i) existence of a threshold for transmission at the excitation wavelength, (ii) an up-conversion excitation threshold, (iii) a long delay for threshold establishment. Such peculiar behaviour had been first observed below $40 \mathrm{~K}$ in $\mathrm{Pr}^{3+}$-doped $\mathrm{LaCl}_{3}$ and $\mathrm{LaBr}_{3}$ crystals $[6,7]$. Soon afterwards, the avalanche pumped laser effect had been obtained in $\operatorname{Pr}^{3+}: \mathrm{LaCl}_{3}$ [8]. Quite recently it has been also observed in $\mathrm{Er}^{3+}: \mathrm{YALO}_{3}[9,10]$ but all of them also below $40 \mathrm{~K}$. Some of the features of the avalanche effect have been observed at room temperature in a $\mathrm{Pr}^{3+}$-doped silica glass fiber [11].

In many cases the initial first step. for avalanche was not considered, the theoretical analysis starting with an already populated metastable state. Such a "by-pass", usually considered for simplification, hides some of the interesting features of the avalanche process, in particular the condition of existence of a meaningful experimental tlireshold.

Analysing the first step of the avalanche effect as an anti-Stokes multiphonon absorption and using the simplified approach of Goldner and Pelle [12], it can 
be shown why this effect is so clearly observed at room temperature in our erbium studies [1-5]. We shall compare our results numerically with other cases of avalanche [10] or with cases which have been called "looping effects" [14] or "quasi-avalanche" ones [15].

\section{The basic photon avalanche process}

While looking for 2-step absorption in $\mathrm{Pr}^{3+}$-doped $\mathrm{LaCl}_{3}$ and $\mathrm{LaBr}_{3}$ at low temperature $(<40 \mathrm{~K})$ as a mean to detect an IR photon by its energy summation with a more energetic photon so performing excited state absorption (ESA), it was found that the higher energy photon alone could, in the same time, give rise to up-conversion and reduce the transmission of the sample above a given intensity threshold [6], see Fig. 1. The effect was attributed to an increase in population on an excited state resulting from a cross-relaxation process. The starting process was initially not completely determined. In the $\mathrm{Pr}^{3+}$ case, the ${ }^{3} \mathrm{H}_{5} \rightarrow{ }^{3} \mathrm{P}_{1}$ absorption is initially very weak at low temperature because ${ }^{3} H_{5}$ is about $2000 \mathrm{~cm}^{-1}$ above the ground state (see Fig. 2); however above about $1 \mathrm{~mW}$ of excitation this transition is increased; the cross-relaxation $\left({ }^{3} H_{6},{ }^{3} H_{4}\right) \rightarrow\left({ }^{3} H_{5},{ }^{3} H_{5}\right)$ increasing the ${ }^{3} H_{5}$ population which in turn reduces the transparency of the sample at the $\left({ }^{3} P_{1}-{ }^{3} H_{5}\right)$ energy. Since the more the $\left({ }^{3} P_{1}-{ }^{3} H_{5}\right)$ energy is absorbed the more the ${ }^{3} H_{5}$ population is increased, the process was termed "photon avalanche" [6]. It is a clear way to increase ESA in a sample.
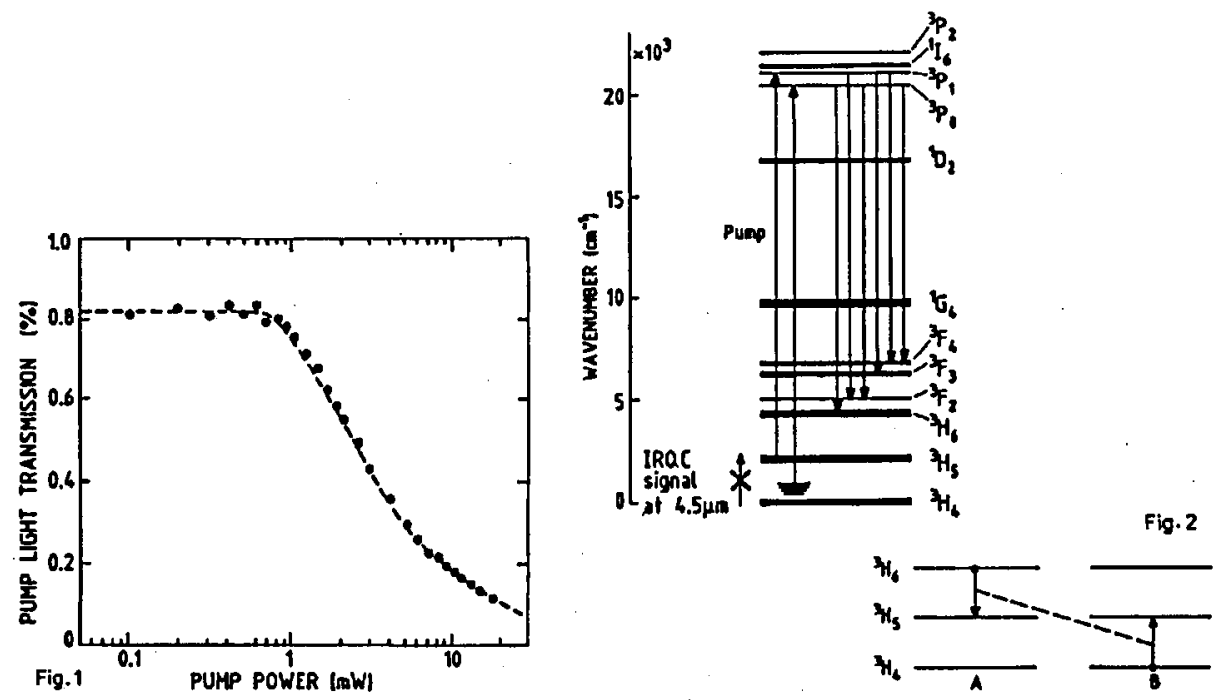

Fig. 1. Decrease of transmission in a $\mathrm{Pr}^{3+}: \mathrm{LaCl}_{3}$ sample under ${ }^{3} H_{5}-{ }^{3} P_{1}$ pumping, after [6].

Fig. 2. $\mathrm{Pr}^{3+}$ energy levels involved in the avalanche process, after [6].

Afterwards, similar effects have been observed in $\mathrm{Sm}^{3+}, \mathrm{Nd}^{3+}, \mathrm{Ni}^{2+}, \mathrm{Tm}^{3+}$ doped halide crystals [16-19]. Recently we obtained the photon avalanche effect at 
room temperature for the $\mathrm{Er}^{3+}$ ion in a $\mathrm{ZBLAN}\left(\mathrm{ZrF}_{4}, \mathrm{BaF}_{2}, \mathrm{LaF}_{3}, \mathrm{AlF}_{3}, \mathrm{NaF}\right)$ glass both in bulk and in fiber form [1-4] and in a LiYF 4 crystal [5].

As already said, there are three distinct aspects for the photon avalanche process non-linear behaviour: (i) transmission, (ii) emission and (iii) rise time on the pump power intensity generally with the existence of a critical pump threshold. served.

Particularly long rise times, from seconds to minutes $[5,20]$ have been ob-

\section{Existence of a threshold}

At this point it is worth discussing the notion of threshold for a valanche. Due to the complexity of the phenomena, it has been usually npdelled by a simplified three-level system $[21,22,12]$

\subsection{The avalanche process as a positive feed-back system}

Using the 3-level simplified model of [21] or [22] and adding to the initial ground state absorption $\left(\sigma_{1} \Phi=R_{1}\right)$ an auxiliary direct feeding into the metastable state $\left(\sigma_{0} \Phi_{I R}\right)$ we may write the following set of equations (see Fig. 3 for explanation of symbols which except for the trigger $\sigma_{0} \Phi_{\mathrm{IR}}$ are the same as in Ref. [21]).

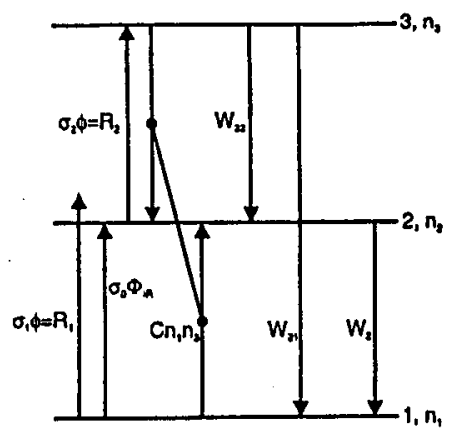

Fig. 3. The a valanche simplified 3-level scheme, $C$ is the cross-relaxation coefficient, $W_{i j}$ are the spontaneous emission terms.

Being interested in the steady state initial step of avalanche we assume

(i) $\frac{\mathrm{d} n_{1}}{\mathrm{~d} t}=\frac{\mathrm{d} n_{2}}{\mathrm{~d} t}=\frac{\mathrm{d} n_{3}}{\mathrm{~d} t}=0$,

(ii) $n_{1}=1-n_{2}-n_{3} \approx 1$.

Then the rate equations are simplified to

$$
\begin{aligned}
& 0=-R_{1}-\sigma_{0} \Phi_{I R}+b W_{3} n_{3}+W_{2} n_{2}-C n_{3}, \\
& 0=(1-b) W_{3} n_{3}-W_{2} n_{2}+2 C n_{3}+R_{1}+\sigma_{0} \Phi_{I R}-R_{2} n_{2}, \\
& 0=R_{2} n_{2}-W_{3} n_{3}-C n_{3}
\end{aligned}
$$

with $C$ the cross-relaxation parameter and $b-$ the branching ratio. The following relationships exist between the transition probabilities:

$$
(1-b) W_{3}=W_{32} ; \quad b W_{3}=W_{31} ; \quad W_{3}=W_{32}+W_{31}
$$


and Eq. (3) can be written as

$$
n_{2} \frac{R_{2}}{W_{3}+C}=n_{3} \text {. }
$$

Or considering amplitude and using the symbolic representation for feed-back systems $\left(3^{\prime}\right)$ give the block A in Fig. 4a.

In the same way Eq. (1) is written as

$$
n_{2}=\frac{R_{1}+\sigma_{0} \Phi_{\mathrm{IR}}}{W_{2}}+\frac{C-b W_{3}}{W_{2}} n_{3},
$$

which can be symbolised in Fig. 4a by the block $\beta$ and adder with the input source $\left(R_{1}+\sigma_{0} \Phi_{\mathrm{IR}}\right) / W_{2}$.

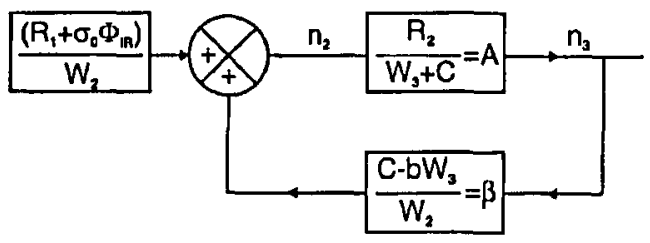

(a)

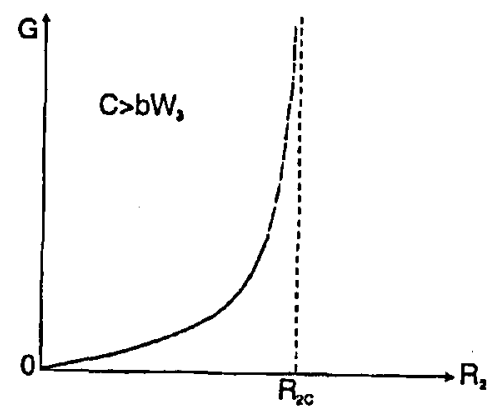

Fig. 4. (a) Positive feed-back model for avalanche. (b) Gain behaviour of the model versus $R_{2}$; positive feed-back condition is $b>C W_{3}$, the asymptote $R_{2 C}$ corresponds to the avalanche threshold.

Combining $\left(1^{\prime}\right)$ and $\left(3^{\prime}\right)$ gives the classical feed-back system scheme in Fig. $4 \mathrm{a}$. Such system is known to be unstable for $A \beta=1$. One can define a "gain" of the closed loop feed-back system, $G$, by the ratio between the green output to a pump related input signal $\left(R_{1}\right)$ plus an eventual trigger $\left(\Phi_{\mathrm{IR}}\right)$

$$
G=\frac{n_{3}}{\left(R_{1}+\sigma_{0} \Phi_{\mathrm{IR}}\right) / W_{2}} .
$$

It is well known that in term of $A$ and $\beta$ in Fig. 4a, one has

$$
G=\frac{A}{1-A \beta} \text {. }
$$

The stability condition is then written as

$$
\frac{R_{2}}{W_{3}+C} \frac{C-b W_{3}}{W_{2}}<1,
$$

its limit is just the threshold condition given by Joubert et al. [21] obtained here in a simplified way 


$$
R_{2 C}=\frac{W_{2}\left(W_{3}+C\right)}{C-b W_{3}}
$$

with $C>b W_{3}$ for a positive feed-back.

The behaviour of our feed-back system below the threshold can be described by the behaviour of $G\left(R_{2}\right)$, Fig. $4 \mathrm{~b}$.

The feed-back "black box" approach has been also considered while studying the dynamics of the above three-level system [23]. It is based on the fact that the general feed-back linear theory used to solve algebraically time variable differential equation systems by using the Laplace transform of the time dependent functions.

\subsection{Conditions to observe a threshold}

With neglect of the first non-resonant absorption step $\left(R_{1}\right)$ and taking into account only the second resonant absorption step $\left(R_{2}\right)$ the calculation of the population of the third level $\left(n_{3}\right)$ versus $R_{2}$ (the pumping excitation) leads to a well-defined non-linearity in $n_{3}$; the asymptotic curve in Fig. 5.

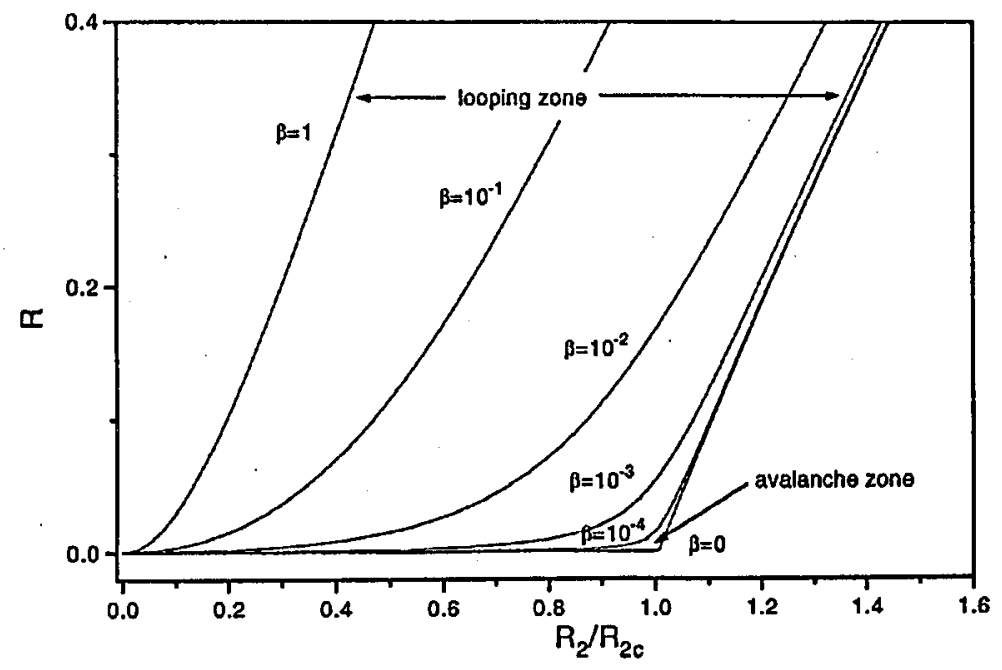

Fig. 5. The third level normalised population, $R=n_{3}\left(\beta, R_{2}\right) / n_{3}\left(\beta=1, R_{2}=R_{2 C}\right)$, versus pumping term $\left(R_{2}\right)$ with $\beta$ as a parameter, after [12].

When the first step $\left(R_{1}\right)$ is explicitly taken into account [12], the "threshold" non-linearity is progressively smoothed out when the ratio $\beta=R_{1} / R_{2}$ is increased as shown in Fig. 5 . This corresponds to a progressively more resonant first step. Practically a clear avalanche threshold can be expected only for $\beta$ ratios $\leq 10^{-4}[12]$.

Some of the features of the avalanche effect have been observed at room temperature in $\mathrm{Tm}^{3+}: \mathrm{YAlO}_{3}[19]$ and in $\mathrm{Pr}^{3+}$ in silica glass fibres [11]. The lack of a clear threshold in this two systems can certainly be related to the above prediction. 
The region in Fig. 5, where $10^{-4}<\beta<1$, corresponds to cases for which the losses in the feed-back loop may exceed the loop gain for $R_{2}$ values below $R_{2 C}$, so that after a number of "looping" of the excited population between level 3 and the metastable level 2 , the system would not diverge and maintain $n_{3}$ independently of $R_{1}$. Such cases have been called "looping mechanism" [14] and we believe that some of the reported cases of quasi-zero threshold avalanche cases in the literature $[11,15]$ belong to large $10^{-3}<\beta \leq 1$ cases for which, as shown in Fig. 5 , it is very difficult to distinguish between avalanche and sequential two-photon absorption (ESA). Sometimes they have been termed "quasi-avalanche" [12].

Up to now, only $\mathrm{Er}^{3+}$ with $\beta \cong 10^{-6}$ has shown, at room temperature, all the three characteristic features of avalanche as well when doping a $\mathrm{LiYF}_{4}$ crystal or a ZBLAN fluoride glass both in bulk and in a fibre shape (see Sec. 4); even the long delay of several seconds to minute was observed [1-5]. For comparison the following values for the critical parameter have been given: for $\mathrm{Nd}^{3+}-\mathrm{LiYF}_{4}$, $\beta=1.7 \times 10^{-4}$ for avalanche at $T<40 \mathrm{~K}[17,21]$; for $\mathrm{Tm}^{3+} \mathrm{IIo}^{3+}-\mathrm{Gd}_{2} \mathrm{Ga}_{5} \mathrm{O}_{12}$, $\beta=3.6 \times 10^{-2}$ for the two-ion looping process [24]; for a $\mathrm{Tm}^{3+}$ BIGaZYTZr glass, $\beta=1.2 \times 10^{-2}$ [25] for what was claimed to be "avalanche" at $100 \mathrm{~K}$ [13]. In the last case because the delay reaches only sixteen times [25] the metastable state lifetime $\left(W_{2}^{-1}\right)$, we believe it to be a looping process case. In $\mathrm{Er}^{3+}$, as it shall be possible to see from Secs. 4 and 5 , the avalanche delay reaches $6 \times 10^{2}$ to $10^{4}$ times $W_{2}^{-1}$, respectively, for fluoride glass and crystal.

\section{Experiments in $\mathrm{Er}^{3+}-\mathrm{LiYF}_{4}$}

In the case of $\mathrm{Er}^{3+}$, the first step for photon avalanche has been clearly identified and attributed to anti-Stokes multiphonon side-bands absorption $[1,5]$ (see Fig. 6). Calculating the $\beta$ ratio from mutiphonon absorption, allows one to estimate a value of $5 \times 10^{-6}[4,5]$, as shown in Fig. 7 which displays the multiphonon side-bands absorption in the avalanche excitation region. As observed, such experimental situation provides a clear threshold behaviour in the erbium case [1].

The simple theory of Sec. 3.1. has been verified by experimentally measuring $G\left(R_{2}\right)$. This was done using the following method (see Fig. 8): having obtained a given green output for a pump $R_{2}$ with $\Phi_{I R}=0, R_{2}$ is reduced while increasing $\Phi_{\text {IR }}$ in order to maintain a constant green output. $\Phi_{\text {IR }}$ is an infrared signal at $0.94 \mu \mathrm{m}$ in resonance with the metastable state 2 (here ${ }^{4} I_{11 / 2}$ ) absorbing with a cross-section $\sigma_{0}$.

Each point is obtained after waiting for a steady state. Because of the large ratio for $\sigma_{0} / \sigma_{1}$ this experiment provides a good description of $G\left(R_{2}\right)$, as shown by results in Fig. 9 for three temperatures. We can define an $R_{2 C}$ asymptote only at $300 \mathrm{~K}$ and $220 \mathrm{~K}$, respectively, $120 \mathrm{~mW}$ and $240 \mathrm{~mW}$. At $163 \mathrm{~K}$ one cannot reach asymptote at our maximum a vailable power of $250 \mathrm{~mW}$. So the effect of lowering temperature is essentially to increase $R_{2 C}$.

The part in $R_{2 C}$ which is most sensitive to temperature is $C$ because it is related to phonon energy of only $100 \mathrm{~cm}^{-1}$, whereas $W_{2}$ and $W_{3}$ are related to phonons covering the energy gap below level 3 and 2 , that is energies $>2000 \mathrm{~cm}^{-1}$. However this requires $C$ to be of the same order as $W_{3}$ or $b W_{3}$, otherwise, as long 


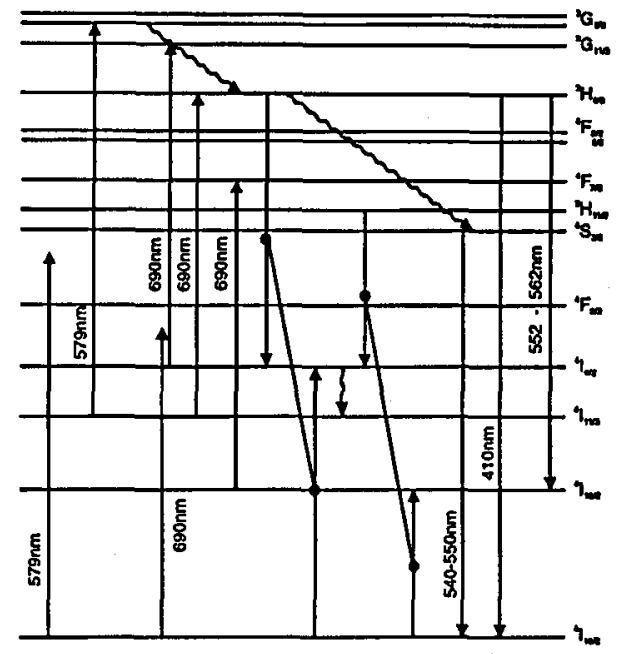

Fig. 6. Energy scheme of $\mathrm{Er}^{3+}$ and principal mechanisms responsible for photon avalanche cycles under excitation at $579 \mathrm{~nm}$ and $690 \mathrm{~nm}$.

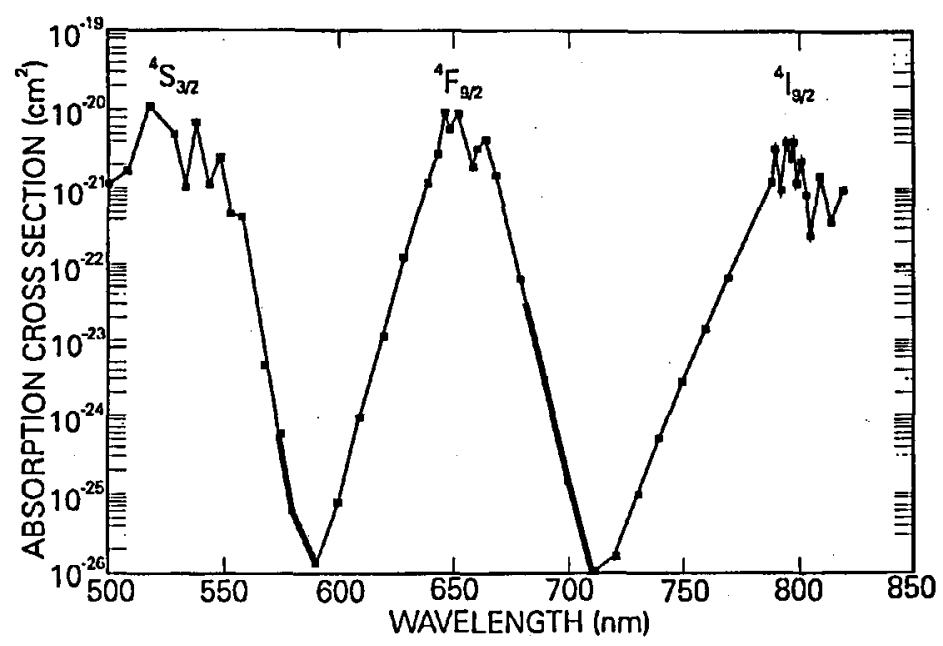

Fig. 7. Absorption cross-section for $\mathrm{Er}^{3+}-\mathrm{LiYF}_{4}$ taking in to account the multiphonon contribution, the heavier lines show the anti-Stokes zones, which contribute to $W_{1}$ for the avalanche processes in erbium, [5].

as $C \gg W_{3}, b W_{3}$, one has $R_{2 C} \cong W_{2}$ and its temperature dependence is just the same as $W_{2}$.

Comparing the theoretical threshold as given by Eq. $\left(6^{\prime}\right)$ with our experimental conditions we can verify our simple model.

We assume level 1 to be ${ }^{4} I_{15 / 2}$, level 2 to be ${ }^{4} I_{11 / 2}$ and level 3 to be the aggregation of levels between ${ }^{2} G_{9 / 2}$ and ${ }^{4} S_{3 / 2}$ with the emission properties of ${ }^{4} S_{3 / 2}$ 


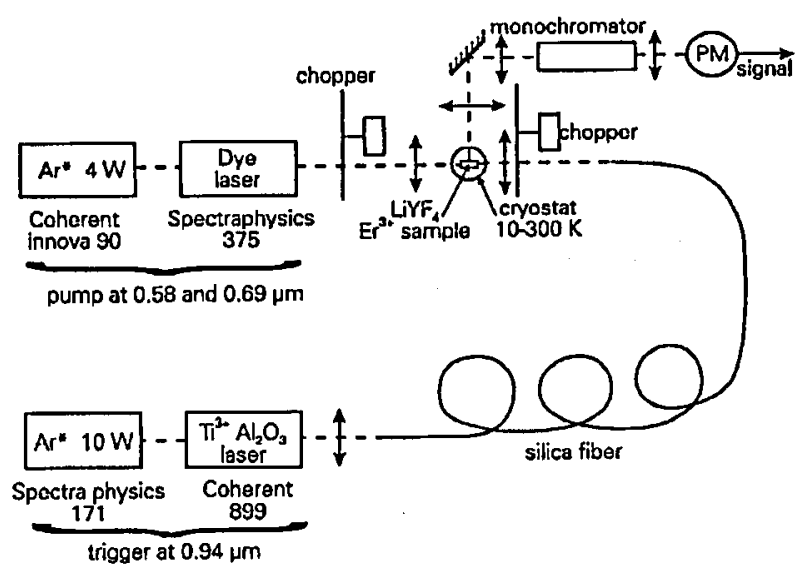

Fig. 8. Experimental scheme for measuring the positive feedrback gain.

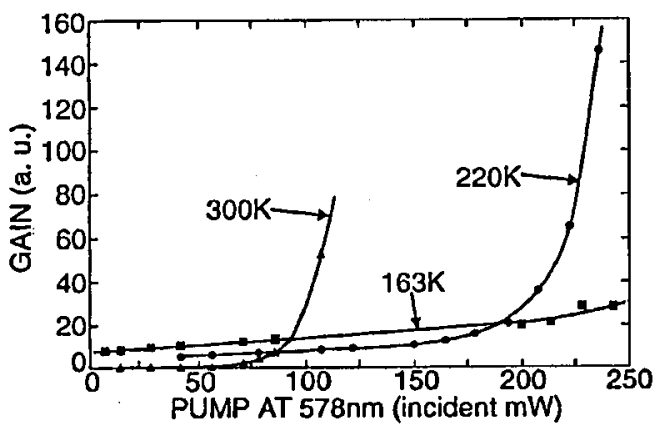

Fig. 9. Experimental $G\left(R_{2}\right)$ for three temperatures: $300 \mathrm{~K}, 220 \mathrm{~K}, 163 \mathrm{~K}$, the residual signal near $R_{2}=0$ comes from the direct up-conversion under $0.94 \mu \mathrm{m}$ excitation, [5].

(see Fig. 6). Taking the room temperature values given by Refs. [26,27] we have the following parameters: $W_{3}=2500 \mathrm{~s}^{-1}, b=0.5, C=0.5 \times 10^{6} \mathrm{~s}^{-1}, W_{2}=140 \mathrm{~s}^{-1}$, because corresponding oscillator strengths are nearly equal $\left(\approx 0.4 \times 10^{-6}\right)$ [27], we assume $\sigma_{2}=\sigma_{0}=4 \times 10^{-21} \mathrm{~cm}^{2}$.

Using the reduced population units (pure number) we have $R_{2 C}=140(5 \times$ $\left.10^{5}+2500\right) /\left(5 \times 10^{5}-1250\right)=141 \mathrm{~s}^{-1} \cong W_{2}$ (at room temperature) from which we have

$$
\Phi_{\text {threshold }}=141 /\left(4 \times 10^{-21}\right)=3.5 \times 10^{22} \mathrm{~s}^{-1} \mathrm{~cm}^{-2},
$$

which at $0.578 \mu \mathrm{m}$ gives for a $50 \mu \mathrm{m}$ diameter spot a threshold power of $P_{\mathrm{th}}=$ $222 \mathrm{~mW}$. This value is of the same order as the observed threshold values for $0.578 \mu \mathrm{m}$ pumping.

$G\left(R_{2}\right)$ shows that we have a "marginally stable" positive feed-back system: even below the $R_{2 C}$ asymptote, we know from the feed-back system behaviour that a strong input signal can drive the system otherwise stable into its instability state (existence of a "gain stability margin"). 
In order to verify this behaviour we experimentally consider the case where, besides the input signal $R_{1} / W_{2}$ given by the pump, we added a pulsed trigger of amplitude $\sigma_{0} \Phi_{\mathrm{IR}} / W_{2}$.

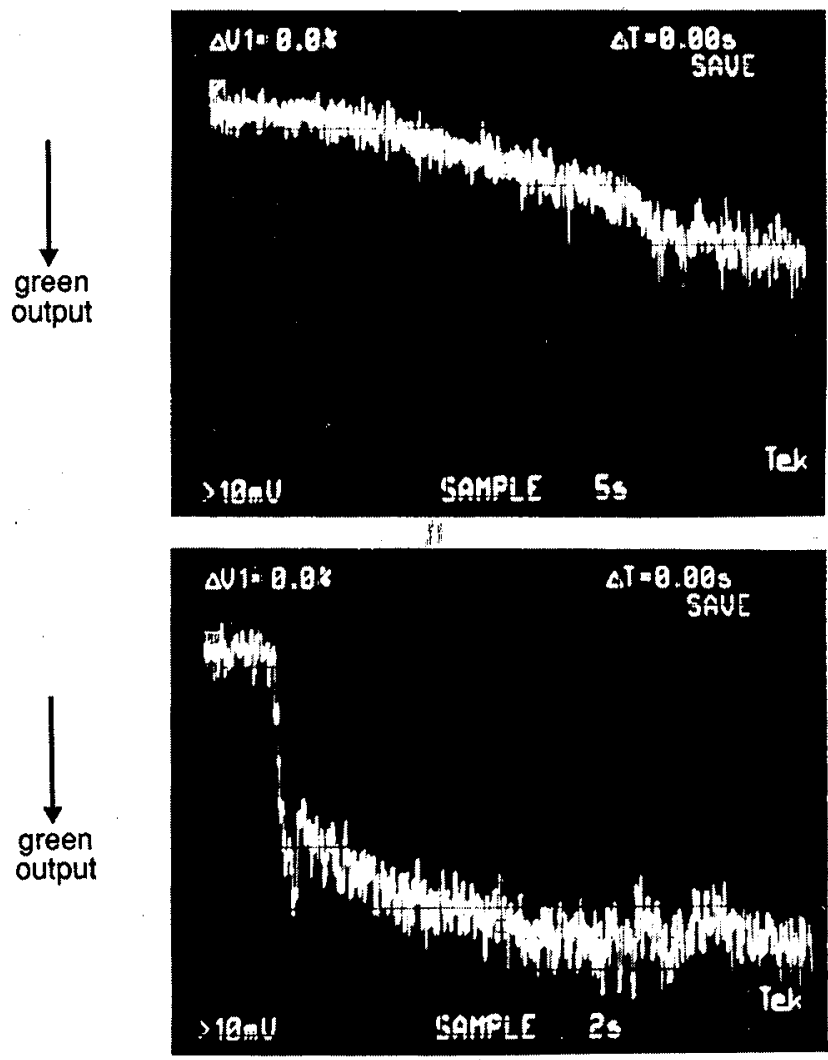

a)

b)

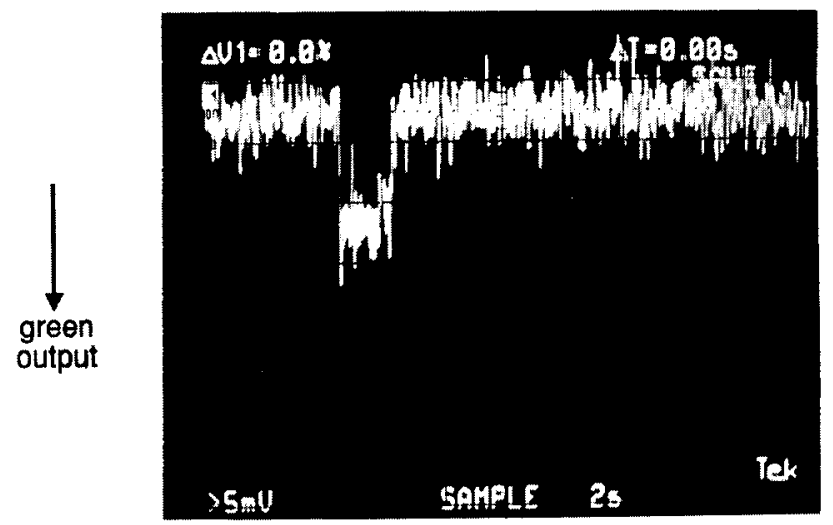

c)

Fig. 10. Avalanche behaviour just below the threshold without (a) and with (b) a trigger feeding the metastable state and (c) much below the threshold, [5]. 
The results at room temperature are given in Fig. 10. In absence of trigger, with $P_{\mathrm{p}}=114 \mathrm{~mW}$ at $578 \mathrm{~nm}$ incident on the sample, the threshold is reached after a very long time ( $>50 \mathrm{~s}$ ) (Fig. 10a). With the same pump intensity $\left(P_{\mathrm{p}}=114 \mathrm{~mW}\right)$ and with a short trigger of $0.6 \mathrm{~s}$, the avalanche state is obtained quickly and maintained after trigger extinction (Fig. 10b). With the same trigger but with a reduced pump $\left(P_{\mathrm{p}}=99 \mathrm{~mW}\right)$ the avalanche state is not reached. The behaviour of Fig. 10a,b,c is obtained down to $180 \mathrm{~K}$.

At temperature lower than $180 \mathrm{~K}$ the observed threshold certainly increases (Fig. 9). However, as our temperature scan has a relatively short time constant ( $3 \mathrm{~s} / \mathrm{K}$ from 10 to $50 \mathrm{~K}$, then $21 \mathrm{~s} / \mathrm{K}$ from 50 to $150 \mathrm{~K}$ ) we are not sure that the threshold could not be reached for an avalanche delay time $\geq 50 \mathrm{~s}$.

From this experiment we understand that measuring a threshold depends on the time we are ready to wait for before its observation and this time depends not only on excited state pumping but also on the ground state absorption conditions. In any case, below $180 \mathrm{~K}$ being then limited by the pump laser at a much lower power than the threshold, we obtain the result shown in Fig. 10c. Such triggering effect realises an optical analogue of a thyratron.

The time delay behaviour of the avalanche process has been studied theoretically quite recently within the general model of Landau for phase transition [25]. The time delay at the threshold, in fact a "critical slowing time", $t_{\mathrm{c}}$, proved itself to be the most sensitive experimental data when looping or avalanche takes place. It has been shown to be given by [25]

$$
t_{\mathrm{c}}=K \sqrt{\sigma_{2} / \sigma_{1}}
$$

where $K$ depends on other spectroscopic parameters. Equation (7) is rather well verified in our experiments for which a valanche delay times have been determined for two different excitation wavelengths of well-known multiphonon anti-Stokes cross-sections: at $\lambda=688 \mathrm{~nm}, \sigma_{1}=10^{-24} \mathrm{~cm}^{2}$, a delay is found to be $0.4 \mathrm{~s}$; whereas at $\lambda=579 \mathrm{~nm}, \sigma_{1}=2 \times 10^{-26}$, and a delay is $4 \mathrm{~s}$. Assuming for $\sigma_{2}$ the same value in both cases of excitation, the ratio of delays is 0.1 which is in rather good accord with the value of 0.14 as given by Eq. (7).

Above the threshold, the delay for avalanche has been given by Ref. [7] as:

$$
t_{\mathrm{c}}=k W_{2}^{-1} /\left(\Phi / \Phi_{C}-1\right)
$$

with $k=W_{3}^{-1} /(1+b)\left(2 c+W_{3}\right)$, and where $\Phi_{C}=R_{C} / \sigma_{2}$ is the pumping flux at the threshold and $\Phi$ - the effective pumping flux.

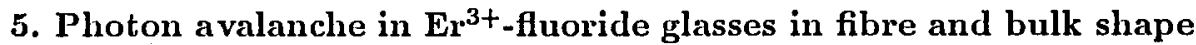

Recently, the photon avalanche effect has been observed in a $\mathrm{Pr}^{3+}$-doped

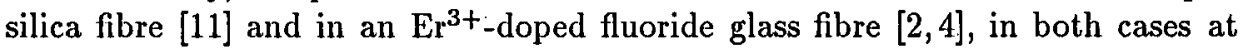
room temperature. In the first case, only the transmission non-linearity is observed and not the up-conversion emission threshold. It was believed that the threshold was so low that it could not be observed. We think that this is explained by the too strong non-resonant to resonant absorption ratio as mentioned in Sec. 3.2. On the opposite in the second case, the clear thresholds at $5 \mathrm{~mW}$ and at $4 \mathrm{~mW}$ of incident power at respectively $579 \mathrm{~nm}$ [4] and $690 \mathrm{~nm}$ [2] are observed because in these last two cases, the first step is a weak anti-Stokes multiphonon absorption 
giving again a $\beta$ ratio of about $10^{-6}$ much below the critical value of $10^{-4}$. The involved energy scheme for both excitations is essentially the same as in Fig. 6; it shows both pumpings and the two types of involved cross-relaxations. Figure 11 presents the typical threshold behaviour for the up-conversion emission. The long delay behaviour is displayed in Fig. 12 showing, near the threshold, the very long time of $3.5 \mathrm{~s}$, which is largely in excess of any of the lifetimes of the metastable states of erbium. The observed delay follows rather well the behaviour predicted by Eq. (8).
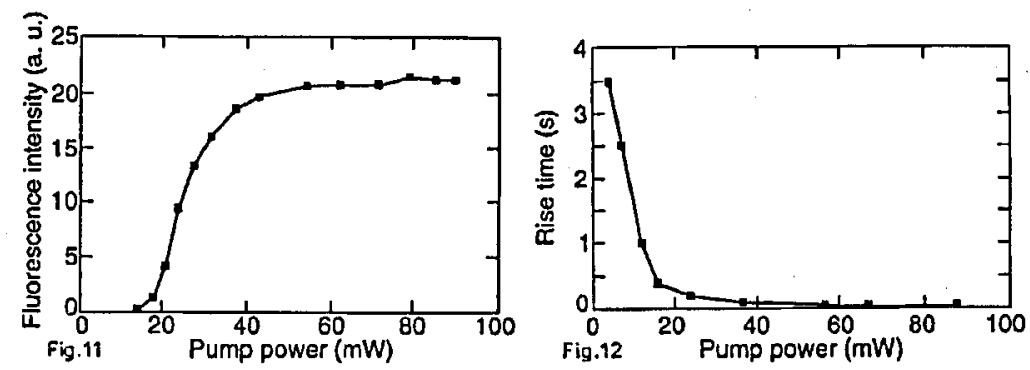

Fig. 11. Up-conversion emission at $550 \mathrm{~nm}$ showing the existence of the avalanche threshold in a ZBLAN: $\mathrm{Er}^{3+}$ fibre observed from its extremity, [4].

Fig. 12. Time delay for the avalanche establishment versus incident pump power at $579 \mathrm{~nm}$ in a ZBLAN: $\mathrm{Er}^{3+}$ fibre, [4].

The same results can be obtained for glass and bulk samples [1,2]. Because the first absorption step, being of a multiphonon nature, is featureless, the excitation spectrum for avalanche provides directly with a single excitation beam the ESA spectrum of the resonant second absorption as shown in Fig. 13 for the ${ }^{4} I_{11 / 2}{ }^{2} G_{9 / 2}$ transition of $\mathrm{Er}^{3+}$. This gives a new method [3] to reach ESA spectra otherwise difficult to obtain.

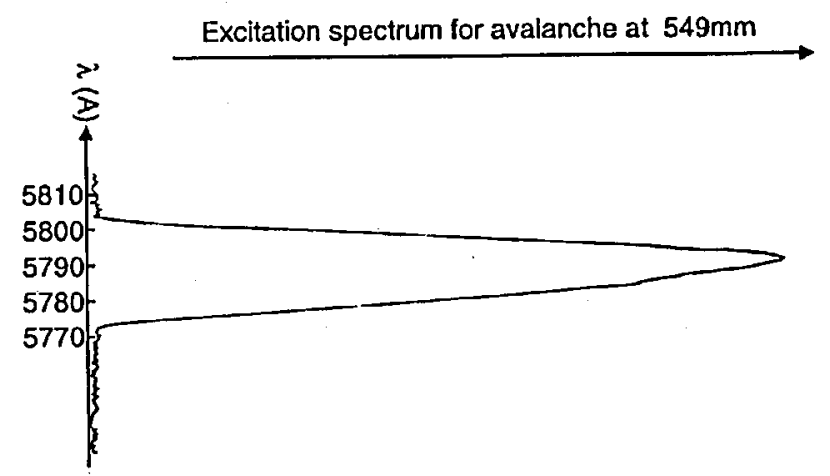

Fig. 13. Excitation spectrum for the avalanche emission at $549 \mathrm{~nm}$ in a ZBLAN: $\mathrm{Er}^{3+}$ glass showing the spectrum for the ${ }^{4} I_{11 / 2}{ }^{2} G_{9 / 2}$ ESA transition, [3]. 

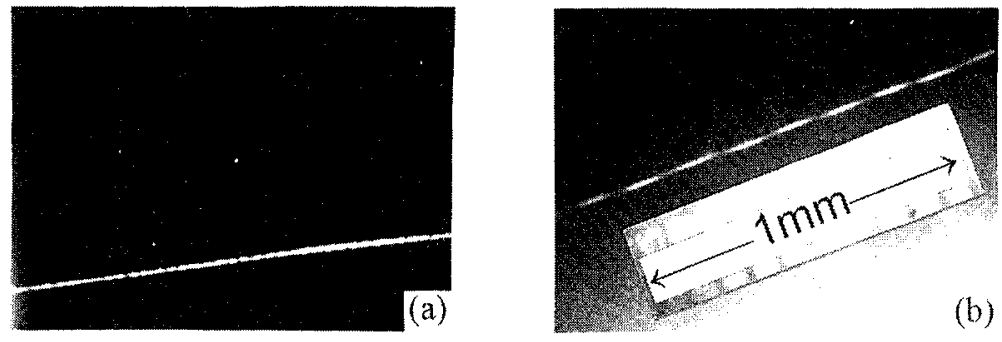

Fig. 14. Spatial domains observed along the fibre above the photon avalanche threshold: (a) dot separation about $1 \mathrm{~mm}$; (b) microscope view of a $1 \mathrm{~mm}$ avalanche dot, the scale is $100 \mu \mathrm{m}$ per division, [2].

The main difference with bulk samples is that in the fluoride fibre case, yet not completely explained, the up-conversion spatial domains appear with periodic structures with periods ranging from few $\mathrm{cm}$ to $\mathrm{mm}$ and $100 \mu \mathrm{m}[2,4]$, as shown in Fig. 14.

\section{Conclusion}

The effective conditions for a clear observation of the rather puzzling photon avalanche effect have been recalled. It has been shown that an erbium ion in fluoride matrices fulfills these conditions so well that this effect is particularly spectacular in such systems even at room temperature. It is striking that a system in the solid state $\left(\mathrm{LiYF}_{4}\right)$ can display a delay of quantum origin as"long as $50 \mathrm{~s}$, corresponding to about $10^{4}$ cycles $\left(t_{c} / W_{2}^{-1}\right)$ in the feed-back loop. Though avalanche pumping of lasers have been demonstrated $[6,7,16]$, and even ASE in $\mathrm{Er}^{3+}$-doped fibre [28], Fig. 5 shows that the population $n_{3}$ is always higher in ESA than in avalanche for the given pumping rate $R_{2}$. This shows that avalanche is not such an effective way of pumping laser except when a double resonance is not readily available for one pumping wavelength.

\section{Acknowledgment}

I would like to thank my former collaborators, Yi Hong Chen and Daniel Meichenin, who have participated actively in the works presented here, and my present collaborators, Fabienne Pellé and Philippe Golner, for making some of their results available prior to publication.

\section{References}

[1] F. Auzel, Y.H. Chen, D. Meichenin, ICL'93, Storrs, CN, USA, 9-12 August 1993; J. Lumin. 60/61, 692 (1994).

[2] Y.H. Chen, F. Auzel, Electron. Lett. 30, 323 (1994).

[3] F. Auzel, Y.H. Chen, J. Non-Crystal. Solids 184, 57 (1995).

[4] Y.H. Chen, F. Auzel, J. Phys. D, Appl. Phys. 28, 207 (1995).

[5] F. Auzel, Y.H. Chen, J. Lumin. 65, 45 (1995).

[6] J.S. Chivian, W.E. Case, D.D. Eden, Appl. Phys. Lett. 35, 124 (1979). 
[7] W.E. Case, M.E. Koch, A.W. Kueny, J. Lumin. 45, 351 (1990).

[8] M.E. Koch, A.W. Kueny, W.E. Case, Appl. Phys. Lett. 56, 1083 (1990).

[9] R. Scheps, IEEE QE30, 2914 (1994).

[10] R. Scheps, IEEE QE31, 309 (1995).

[11] A.S.L. Gomes, G.S. Maciel, R.E. de Araujo, L.H. Acioli, C.B. de Araujo, Opt. Commun. 103, 361 (1993).

[12] P. Goldner, F. Pellé, Optical Materials, to be published, (1995); F. Pellé, P. Goldner, Acte Phys. Pol. A 00, 197 (1996).

[13] M.F. Joubert, S. Guy, C. Linares, B. Jacquier, J.L. Adam, J. Non-Cryst. Solids 184, 98 (1995).

[14] A. Brenier, L.C. Courrol, C. Pédrini, C. Madej, G. Boulon, J. Lumin. 58, 285 (1994).

[15] J.M. Dyson, S.M. Jaffe, II. Eilers, M.L. Jones, W.M. Dennis, W.M. Yen, J. Lumin. 60/61, 668 (1994).

[16] N.J. Krasutsky, J. Appl. Phys. 54, 1261 (1983).

[17] W. Lenth, R.M. Macfarlane, J. Lumin. 45, 346 (1990).

[18] U. Octliker, M.J. Riley, P.S. May, H.U. Güdel, J. Lumin. 53, 553 (1992).

[19] H. Ni, S.C. Rand, Opt. Lett. 17, 1222 (1992).

[20] N. Pelletier-Allard, R. Pelletier, Phys. Rev. B 26, 4425 (1987).

[21] M.F. Joubert, S. Guy, B. Jacquier, Phys. Rev. B 48, 10031 (1993).

[22] A.W. Kueny, W.E. Case, M.E. Koch, J. Opt. Soc. Am. B 10, 1834 (1993).

[23] A. Brenier, G. Boulon, C. Madej, C. Pédrini, L. Lou, J. Lumin. 54, 271 (1993).

[24] A. Brenier, L.C. Courrol, C. Pédrini, C. Madej, G. Boulon, Opt. Mater. 3, 25 (1994).

[25] S. Guy, Ph.D. Thesis, Université Claude-Bernard-Lyon I, 1995.

[26] J. Rubin, A. Brenier, R. Moncorgé, C. Pédrini, J. Lumin. 36, 39 (1986).

[27] S. IIubert, D. Meichenin, B. Zhou, F. Auzel, J. Lumin. 50, 7 (1991).

[28] Y.H. Chen, F. Auzel, Electron. Lett. 30, 1602 (1994). 